\title{
Transverse Energy Measurements from the Beam Energy Scan in PHENIX
}

\author{
J.T. Mitchell (for the PHENIX Collaboration) ${ }^{1}$ \\ Brookhaven National Laboratory, Building 510C, P.O. Box 5000, Upton, NY 11973-5000
}

\begin{abstract}
Transverse energy distributions at midrapidity have been measured by the PHENIX experiment at the BNL Relativistic Heavy Ion Collider (RHIC) for $\mathrm{Au}+\mathrm{Au}, \mathrm{U}+\mathrm{U}, \mathrm{Cu}+\mathrm{Au}, \mathrm{Cu}+\mathrm{Cu},{ }^{3} \mathrm{He}+\mathrm{Au}, d+\mathrm{Au}$, and $p+p$ collisions over a wide energy range from $\sqrt{s_{N N}}=7.7 \mathrm{GeV}$ to $\sqrt{s_{N N}}=200 \mathrm{GeV}$ as a function of centrality. For central Au+Au collisions, it is observed that the midrapidity Bjorken energy density demonstrates a power law behavior from $\sqrt{s_{N N}}=7.7 \mathrm{GeV}$ to $\sqrt{s_{N N}}=2.76$ $\mathrm{TeV}$. At a given collision energy, the data presented as a function of $N_{\text {part }}$ are independent of the size of the collision system. For $\mathrm{Au}+\mathrm{Au}, \mathrm{Cu}+\mathrm{Au}$, and $\mathrm{Cu}+\mathrm{Cu}$ collisions, the centrality-dependent data are better described by scaling with the number of constituent quark participants than scaling with the number of nucleon participants.
\end{abstract}

Keywords:

\section{Introduction}

The PHENIX experiment at the BNL Relativistic Heavy Ion Collider (RHIC) has compiled a comprehensive dataset from the years 2000 to 2015 that includes collisions of $\mathrm{Au}+\mathrm{Au}, \mathrm{U}+\mathrm{U}, \mathrm{Cu}+\mathrm{Au}, \mathrm{Cu}+\mathrm{Cu}$, ${ }^{3} \mathrm{He}+\mathrm{Au}, d+\mathrm{Au}$, and $p+p$ collisions at a variety of collision energies. This dataset can be exploited to study the dynamics of the colliding system with measurements of transverse energy production at midrapidity, $d \mathrm{E}_{T} / d \eta$, as a function of collision energy and centrality. This study is complementary and extends previous studies of dynamics based on charged particle multiplicity production by the PHOBOS experiment [1]. Although PHENIX has also measured charged particle multiplicity [2, 3], only transverse energy measurements will be presented in this article. More details on the PHENIX transverse energy measurements presented here can be found elsewhere [2, 4, 3].

\section{Energy Dependence of Transverse Energy Production}

Figure 1(left) shows transverse energy production, $\left(d \mathrm{E}_{T} / d \eta\right) /\left(0.5 N_{\text {part }}\right)$, for central $\mathrm{Au}+\mathrm{Au}$ and $\mathrm{Pb}+\mathrm{Pb}$ collisions at midrapidity as a function of $\sqrt{s_{N N}}$. Above $\sqrt{s_{N N}}=7.7 \mathrm{GeV}$, the value of $\left(d \mathrm{E}_{T} / d \eta\right) /\left(0.5 N_{\text {part }}\right)$

\footnotetext{
${ }^{1}$ For the full PHENIX Collaboration author list and acknowledgements, see Appendix "Collaboration" of this volume.
} 
is observed to be well described by a power law, $\left(d \mathrm{E}_{T} / d \eta\right) /\left(0.5 N_{\text {part }}\right) \propto \sqrt{s_{N N}}$, where the exponent is $b=0.428 \pm 0.021$. These results can also be expressed in terms of the Bjorken energy density [5]

$$
\varepsilon_{B J}=\frac{1}{A_{\perp} \tau} \frac{d \mathrm{E}_{T}}{d \eta}
$$

where $A_{\perp}$ is the transverse overlap area of the nuclei determined from the Glauber model and $\tau$ is the formation time, typically estimated to be $1 \mathrm{fm} / \mathrm{c}$. Figure 1 (right) shows the Bjorken energy density multiplied by the formation time for central $\mathrm{Au}+\mathrm{Au}$ and $\mathrm{Pb}+\mathrm{Pb}$ collisions above $\sqrt{s_{N N}}=7.7 \mathrm{GeV}$. These data are well described by $\varepsilon_{B J} \tau \propto{\sqrt{s_{N N}}}^{b}$, where $b=0.422 \pm 0.035$.
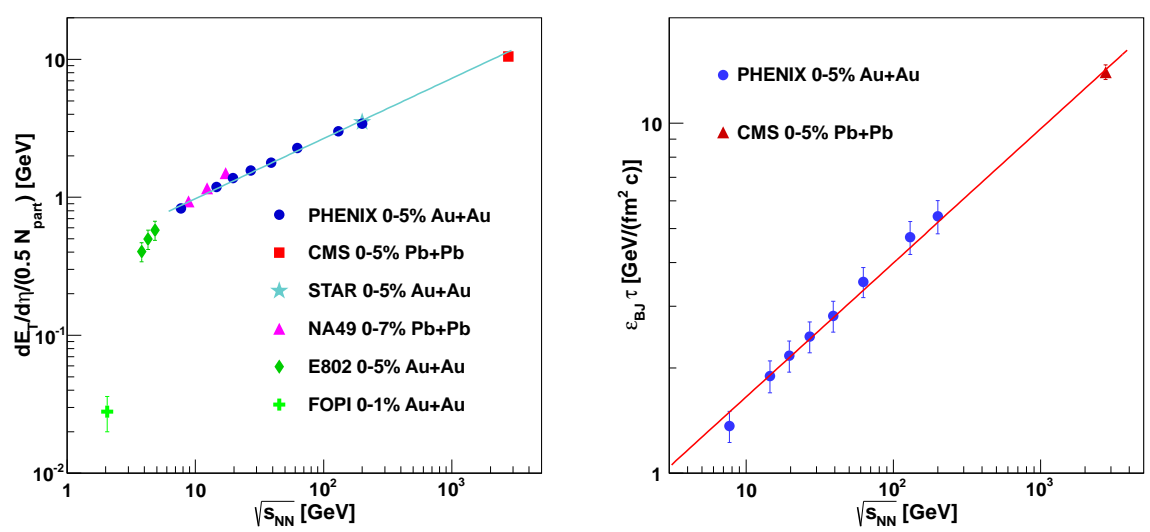

Fig. 1. (Left) $\left(d \mathrm{E}_{T} / d \eta\right) /\left(0.5 N_{\text {part }}\right)$ as a function of $\sqrt{s_{N N}}$ for central $\mathrm{Au}+\mathrm{Au}$ and $\mathrm{Pb}+\mathrm{Pb}$ collisions. In addition to the PHENIX data, data are shown from FOPI [6], E802 [7], NA49 [8], STAR [9], and CMS [10]. The line is a power law fit to the PHENIX data. (Right) $\varepsilon_{B J}$ multiplied by $\tau$ as a function of $\sqrt{S_{N N}}$ for central $\mathrm{Au}+\mathrm{Au}$ and $\mathrm{Pb}+\mathrm{Pb}$ collisions. In addition to the PHENIX data, data are shown from CMS [10]. The line is a power law fit to all of the data points.

\section{System Size Dependence of Transverse Energy Production}

The PHENIX dataset includes $\mathrm{Au}+\mathrm{Au}, \mathrm{Cu}+\mathrm{Au}$, and $\mathrm{Cu}+\mathrm{Cu}$ collisions at $\sqrt{s_{N N}}=200 \mathrm{GeV}$ along with $\mathrm{Au}+\mathrm{Au}$ and $\mathrm{Cu}+\mathrm{Cu}$ collisions at $\sqrt{s_{N N}}=62.4 \mathrm{GeV}$. This facilitates a study of the system size dependence of transverse energy production. Figure 2 shows the Bjorken energy density multiplied by the formation time as a function of $N_{\text {part }}$ for these systems. At a given collision energy, $\varepsilon_{B J}$ for systems of differing sizes are consistent with each other. This demonstrates that $\varepsilon_{B J}$ is independent of the system size at $\sqrt{S_{N N}}=200$ and $62.4 \mathrm{GeV}$.

\section{Centrality Dependence of Transverse Energy Production}

The centrality dependence of $\left(d \mathrm{E}_{T} / d \eta\right) /\left(0.5 N_{\text {part }}\right)$ is typically expressed in terms of the number of nucleon participants, $N_{\text {part }}$, as shown in Figure 3 (left) for Au+Au collisions from $\sqrt{s_{N N}}=7.7$ to $200 \mathrm{GeV}$. Note that the midrapidity data increase with increasing $N_{\text {part }}$ and are not consistent with scaling by the number of nucleon participants in $\mathrm{Au}+\mathrm{Au}$ collisions from $\sqrt{S_{N N}}=200 \mathrm{GeV}$ all the way down to $\sqrt{S_{N N}}=7.7 \mathrm{GeV}$. The data can also be examined as a function of centrality expressed as the number of constituent quark participants, $N_{\mathrm{qp}}[11]$. This has been estimated using a Glauber model calculation that has been modified to replace nucleons with their constituent quarks [3]. The results are shown in Figure 3](right), which shows $\left(d \mathrm{E}_{T} / d \eta\right) /\left(0.5 N_{\mathrm{qp}}\right)$ as a function of $N_{\mathrm{qp}}$ for $\mathrm{Au}+\mathrm{Au}$ collisions from $\sqrt{s_{N N}}=200 \mathrm{GeV}$ down to $7.7 \mathrm{GeV}$. For all energies, the data are better described by scaling with $N_{\mathrm{qp}}$ than scaling with $N_{\text {part }}$. This is consistent with 


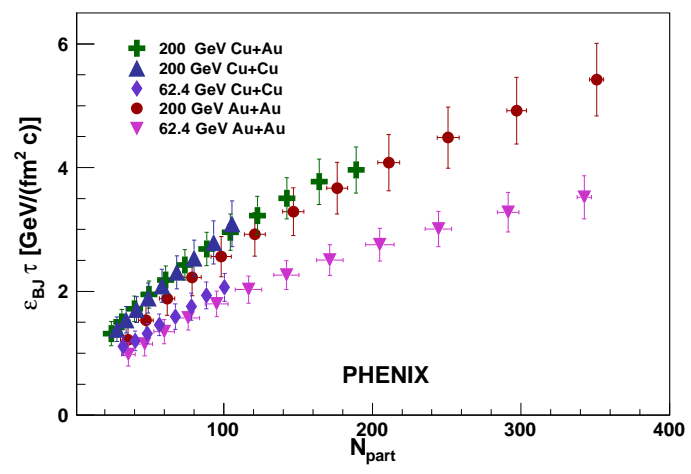

Fig. 2. $\varepsilon_{B J}$ as a function of $\sqrt{s_{N N}}$ for $\mathrm{Au}+\mathrm{Au}, \mathrm{Cu}+\mathrm{Au}$, and $\mathrm{Cu}+\mathrm{Cu}$ collisions at $\sqrt{s_{N N}}=200$ and $62.4 \mathrm{GeV}$. The error bars represent the statistical and systematic errors.

previous studies of charged particle multiplicity distributions measured by PHOBOS down to $\sqrt{s_{N N}}=19.6$ $\mathrm{GeV}$ [12]. Figure 4 shows $\left(d \mathrm{E}_{T} / d \eta\right) /\left(0.5 N_{\mathrm{qp}}\right)$ as a function of $N_{\mathrm{qp}}$ for $\mathrm{Cu}+\mathrm{Au}$ and $\mathrm{Cu}+\mathrm{Cu}$ collisions at $\sqrt{S_{N N}}$ $=200$ and $62.4 \mathrm{GeV}$. The data are also better described by scaling with $N_{\mathrm{qp}}$ for the smaller systems.
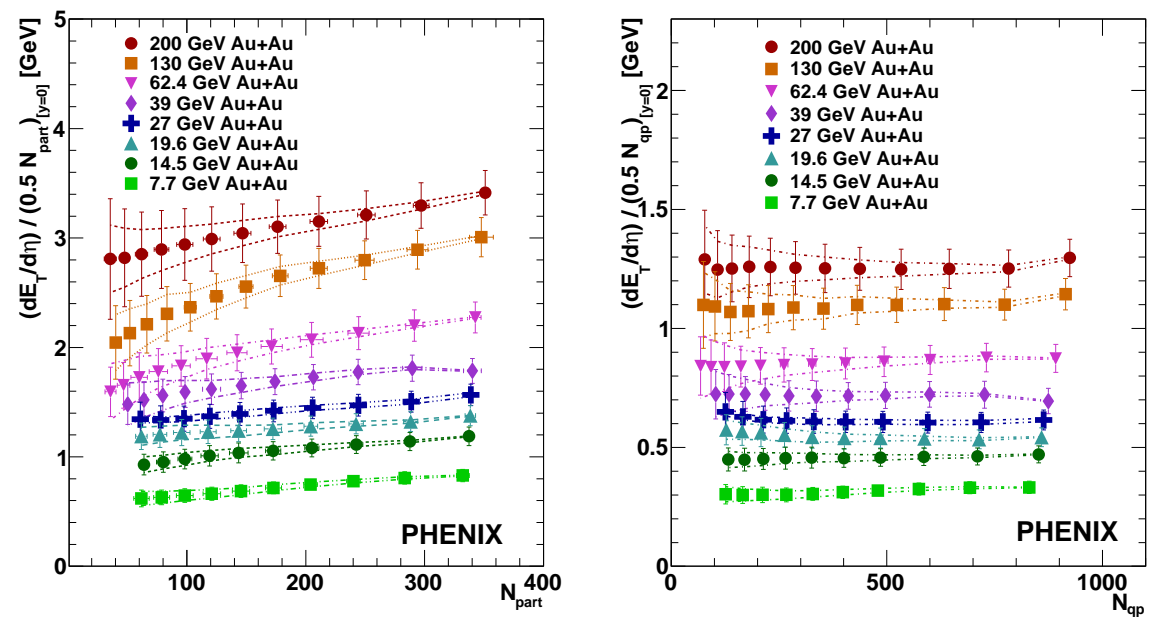

Fig. 3. (Left) $\left(d \mathrm{E}_{T} / d \eta\right) /\left(0.5 N_{\text {part }}\right)$ as a function of $N_{\text {part }}$ for $\mathrm{Au}+\mathrm{Au}$ collisions from $\sqrt{s_{N N}}=200 \mathrm{GeV}$ to $7.7 \mathrm{GeV}$. (Right) $\left(d \mathrm{E}_{T} / d \eta\right) /\left(0.5 N_{\mathrm{qp}}\right)$ as a function of $N_{\mathrm{qp}}$ for $\mathrm{Au}+\mathrm{Au}$ collisions from $\sqrt{s_{N N}}=200 \mathrm{GeV}$ to $7.7 \mathrm{GeV}$. For both plots, the lines bounding the points represent the trigger efficiency uncertainty within which the points can be tilted. The error bars represent the remaining statistical and systematic errors.

\section{Summary}

The PHENIX experiment has completed a systematic survey of transverse energy production in a variety of collision systems for $\sqrt{s_{N N}}=7.7 \mathrm{GeV}$ to $\sqrt{s_{N N}}=200 \mathrm{GeV}$. The Bjorken energy density for central Au+Au collisions at midrapidity is well described by a power law from $\sqrt{s_{N N}}=7.7 \mathrm{GeV}$ up to $\sqrt{s_{N N}}=2.76 \mathrm{TeV}$. At $\sqrt{S_{N N}}=200$ and $62.4 \mathrm{GeV},\left(d \mathrm{E}_{T} / d \eta\right) /\left(0.5 N_{\text {part }}\right)$ presented as a function of $N_{\text {part }}$ is independent of the size of the system. For $\sqrt{S_{N N}}=7.7$ to $200 \mathrm{GeV}$, it is observed that transverse energy production is better 


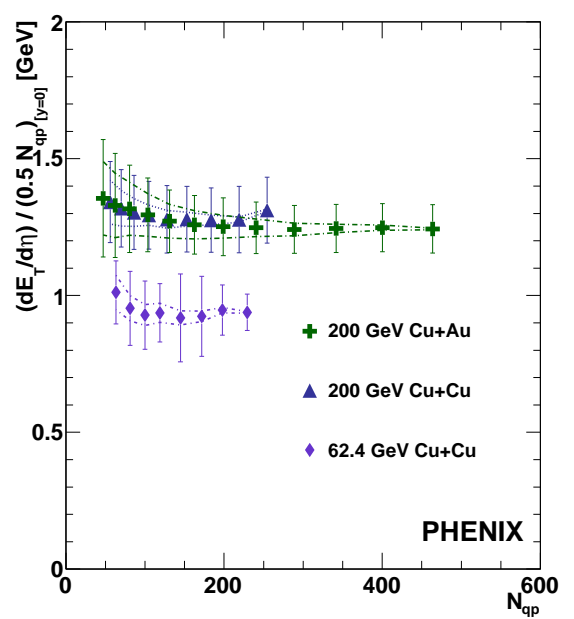

Fig. 4. $\left(d \mathrm{E}_{T} / d \eta\right) /\left(0.5 N_{\mathrm{qp}}\right)$ as a function of $N_{\mathrm{qp}}$ for $\mathrm{Cu}+\mathrm{Au}$ and $\mathrm{Cu}+\mathrm{Cu}$ collisions for $\sqrt{s_{N N}}=200 \mathrm{GeV}$ and $\sqrt{s_{N N}}=62.4 \mathrm{GeV}$. The lines bounding the points represent the trigger efficiency uncertainty within which the points can be tilted. The error bars represent the remaining statistical and systematic errors.

described by scaling with respect to the number of constituent quark participants than by the number of nucleon participants.

\section{References}

[1] B. Alver, et al., Phobos results on charged particle multiplicity and pseudorapidity distributions in Au+Au, $\mathrm{Cu}+\mathrm{Cu}, \mathrm{d}+\mathrm{Au}$, and $\mathrm{p}+\mathrm{p}$ collisions at ultra-relativistic energies, Phys.Rev. C83 (2011) 024913. arXiv:1011.1940. doi:10.1103/PhysRevC.83.024913

[2] S. Adler, et al., Systematic studies of the centrality and $s(\mathrm{NN}) * *(1 / 2)$ dependence of the $\mathrm{d} E(\mathrm{~T}) / \mathrm{d}$ eta and $\mathrm{d}$ (N(ch) / d eta in heavy ion collisions at mid-rapidity, Phys.Rev. C71 (2005) 034908. arXiv:nucl-ex/0409015. doi:10.1103/PhysRevC.71.049901,10.1103/PhysRevC.71.034908

[3] A. Adare, et al., Transverse energy production and charged-particle multiplicity at midrapidity in various systems from $\sqrt{s_{N N}}=$ 7.7 to $200 \mathrm{GeVarXiv:1509.06727}$

[4] S. Adler, et al., Transverse-energy distributions at midrapidity in $\mathrm{p}+\mathrm{p}, \mathrm{d}+\mathrm{Au}$, and $\mathrm{Au}+\mathrm{Au}$ collisions at $\sqrt{s_{N N}}=$ $62.4200 \mathrm{GeV}$ and implications for particle-production models, Phys.Rev. C89 (4) (2014) 044905. arXiv:1312.6676. doi:10.1103/PhysRevC.89.044905

[5] J. Bjorken, Highly Relativistic Nucleus-Nucleus Collisions: The Central Rapidity Region, Phys.Rev. D27 (1983) $140-151$. doi:10.1103/PhysRevD.27.140

[6] W. Reisdorf, et al., Central collisions of $\mathrm{Au}$ on $\mathrm{Au}$ at 150, 250 and $400 \mathrm{MeV} /$ nucleon, Nucl.Phys. A612 (1997) $493-556$. arXiv:nucl-ex/9610009 doi:10.1016/S0375-9474(96)00388-0

[7] L. Ahle, et al., Simultaneous multiplicity and forward energy characterization of particle spectra in Au + Au collisions at 11.6A-GeV/c, Phys.Rev. C59 (1999) 2173-2188. doi:10.1103/PhysRevC.59.2173

[8] S. Afanasiev, T. Anticic, B. Baatar, D. Barna, J. Bartke, et al., Recent results on spectra and yields from NA49, Nucl.Phys. A715 (2003) 161-170. arXiv:nucl-ex/0208014 doi:10.1016/S0375-9474(02)01424-0

[9] J. Adams, et al., Measurements of transverse energy distributions in Au + Au collisions at s(NN)**(1/2) $=200-\mathrm{GeV}, \mathrm{Phys} . \mathrm{Rev}$. C70 (2004) 054907. arXiv:nucl-ex/0407003 doi:10.1103/PhysRevC.70.054907

[10] S. Chatrchyan, et al., Measurement of the pseudorapidity and centrality dependence of the transverse energy density in $\mathrm{PbPb}$ collisions at $\sqrt{s_{N N}}=2.76 \mathrm{TeV}$, Phys.Rev.Lett. 109 (2012) 152303. arXiv:1205.2488. doi:10.1103/PhysRevLett.109.152303

[11] S. Eremin, S. Voloshin, Nucleon participants or quark participants?, Phys.Rev. C67 (2003) 064905. arXiv:nucl-th/0302071. doi:10.1103/PhysRevC.67.064905

[12] R. Nouicer, Charged particle multiplicities in A+A and $p^{+} p$ collisions in the constituent quarks framework, Eur.Phys.J. C49 (2007) 281-286. arXiv:nucl-th/0608038 doi:10.1140/epjc/s10052-006-0128-z 


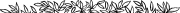

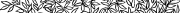

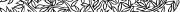
twa

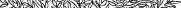

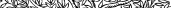

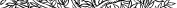
W

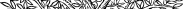
Lar

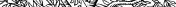
if

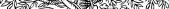

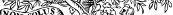

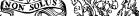

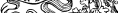

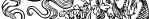

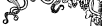

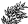
intary

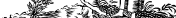

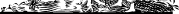

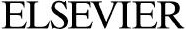

